\title{
Rôle du secteur de la santé publique face à la maltraitance des enfants au Canada
}

\author{
S. M. Jack, R.N., Ph. D. (1)
}

\section{Résumé}

La maltraitance des enfants est un problème de santé et de société important, compte tenu de sa prévalence dans la population générale et de ses effets pervers à court et à long termes. Une approche intégrée, concertée et plurisectorielle s'impose pour circonscrire, prévenir et contrer ce problème complexe. Dans le cadre de cette approche plurisectorielle, le secteur de la santé publique au Canada doit impérativement définir son rôle dans les mesures de lutte et de prévention. Nous présentons, dans ce commentaire, la façon dont le secteur de la santé publique pourrait aborder ce problème au Canada, à savoir : 1) déterminer l'ampleur du problème au moyen de systèmes de surveillance de la santé publique telle l'Étude canadienne sur l'incidence des signalements de cas de violence et de négligence envers les enfants; 2) circonscrire les facteurs de risque modifiables; 3 ) cerner et évaluer les interventions de proximité visant à prévenir la violence; 4) mettre en œuvre des stratégies de prévention primaire qui s’appuient sur des preuves.

Mots clés : prévention primaire, santé publique, violence envers les enfants, intervention, visites à domicile d'infirmières, partenariat infirmières-familles, maltraitance d'enfants, surveillance

\section{Introduction}

La maltraitance des enfants suppose qu'un préjudice a été ou risque d'être causé à un enfant ou à un jeune par un adulte en qui il a confiance ou dont il dépend ${ }^{1}$. Ce préjudice peut résulter d'un acte de commission (par exemple, brutalités physiques, exploitation sexuelle ou violence psychologique) ou d'un acte d'omission (par exemple, négligence physique, psychologique ou médicale, omission de superviser ou exposition à la violence) ${ }^{1}$. Les mauvais traitements subis pendant l'enfance sont associés à des troubles physiques, sociaux, psychologiques et cognitifs à court et à long termes qui peuvent laisser des séquelles permanentes $^{2,3}$. L'exposition aux mauvais traitements pendant l'enfance est fréquente : environ le tiers des adultes canadiens disent avoir été l'objet de violence physique ou de sévices sexuels (ou les deux) pendant leur enfance ${ }^{4}$.

La maltraitance des enfants peut être difficile à circonscrire, car il n'y a pas de consensus entre les gouvernements et les secteurs (services de santé, droit, enseignement, justice) sur les « actes » qui constituent un abus. En tant que problème de santé et de société, la maltraitance des enfants est difficile à prévenir, car il existe des indicateurs de risque aux niveaux individuel, familial et collectif. Il est également difficile d'intervenir contre la maltraitance des enfants ou de la traiter, puisque les différents secteurs ont des rôles et des responsabilités distincts à ce chapitre. Face à cette situation complexe, il y aurait lieu d'adopter une approche intégrée, concertée et plurisectorielle pour circonscrire, prévenir et intervenir.

Bien que tous les professionnels des différents secteurs soient tenus de déclarer les cas présumés ou observés de maltraitance d'enfants aux services d'aide à l'enfance, chaque secteur a sa façon propre d'opérer (tableau 1). Il est essentiel que le secteur de la santé publique canadien définisse son rôle face à la maltraitance des enfants dans le cadre de cette approche plurisectorielle, car c'est lui qui fait le lien entre les déterminants biologiques et individuels des troubles de la santé et les déterminants sociaux, économiques et politiques qui influent sur la santé des populations ${ }^{5}$.

\section{Aborder la maltraitance des enfants dans une optique de santé publique}

Dans ce commentaire, nous indiquons comment le problème de la maltraitance des enfants pourrait être abordé sous l'angle de la santé publique, à savoir: 1) déterminer l'ampleur du problème au moyen de systèmes de surveillance de la santé publique; 2) circonscrire les facteurs de risque modifiables; 3 ) cerner et évaluer les interventions de proximité visant à prévenir la violence; 4) mettre en œuvre des stratégies de prévention primaire qui s'appuient sur des données probantes. 


\section{Aborder les problèmes dans une optique de santé publique}

La résolution des problèmes dans une optique de santé publique $^{5-7}$ comporte quatre étapes distinctes : 1) mesurer l'ampleur du problème au moyen de méthodes de surveillance et de méthodes épidémiologiques; 2) définir les causes et les corrélats du problème, dont les indicateurs de risque ou de protection pouvant être modifiés au moyen de mesures d'intervention ou de prévention; 3) élaborer et mettre en œuvre des interventions, puis les évaluer; 4) mettre en œuvre les interventions dont l'influence sur des paramètres pertinents et cliniquement significatifs a été démontrée.

\section{Mesure de la maltraitance des enfants au Canada}

Au niveau fédéral, l'Agence de la santé publique du Canada (ASPC) définit et mesure l'incidence et les facteurs de risque de la maltraitance des enfants et les effets que les enquêtes sur les cas de maltraitance ont sur les services. Il apparaît maintenant prioritaire de faire en sorte que ces données soient accessibles aux décideurs de la santé publique des provinces, des territoires et des régions chargés de circonscrire les problèmes de santé publique et de mettre en œuvre des programmes de prévention primaire. Dans le domaine de la santé publique, l'ampleur d'un problème est mesurée au moyen de données épidémiologiques et de surveillance. C'est dans ce cadre que l'ASPC coordonne l'Étude canadienne sur l'incidence des signalements de cas de violence et de négligence envers les enfants $(\mathrm{ECI})^{8}$, une étude nationale de surveillance de la santé publique réalisée tous les cinq ans en vue de recueillir des données sur cinq catégories de mauvais traitements envers les enfants, depuis la naissance jusqu'à l'âge de 15 ans : négligence, violence psychologique, exposition à la violence familiale, sévices sexuels et violence physique. À l'automne 2003, des travailleurs des services de protection de l'enfance ont recueilli des données pour l'ECI 2003 auprès d'un échantillon représentatif de 63 régions de services d'aide à l'enfance dans l'ensemble du Canada ayant suivi un total de 14200 enquêtes sur des cas de maltraitance d'enfants.
Au Québec, les données ont été extraites à partir d'un fichier administratif; dans les autres provinces et dans les territoires, les travailleurs de la protection de l'enfance ont rempli un formulaire d'évaluation normalisée ${ }^{8}$ Des données ont été recueillies sur les caractéristiques des enfants visés par les enquêtes, les détails des enquêtes, le degré de justification, les conséquences sur la santé de l'enfant, les mesures prises à court terme pour soustraire l'enfant aux actes de maltraitance et les caractéristiques de la famille et du ménage.

Or, Pless avance que les systèmes de surveillance de la santé publique sont souvent centrés sur la collecte et l'analyse consciencieuses de données et que les conclusions obtenues sont rarement transmises en temps opportun aux décideurs concernés et désireux d'agir dans la mise en œuvre des programmes de prévention`. Sa conclusion est que «l'idéal serait de faire en sorte que la surveillance serve les objectifs de la prévention. " Bien que l'ECI soit coordonnée par l'intermédiaire de l'ASPC, ses résultats sont prioritairement communiqués au grand public et aux décideurs du domaine de l'aide à l'enfance $^{10}$ ayant pour mandat d'identifier les enfants victimes de mauvais traitements et de mettre en œuvre des programmes de prévention secondaire afin de réduire les taux de récidive et les troubles de la santé associés à la maltraitance ${ }^{11}$. Pour aider à la planification, à la mise en œuvre et à l'évaluation de programmes de prévention primaire, il faudrait que les données de surveillance de l'ECI soient communiquées aux décideurs et aux chercheurs des organismes de santé publique responsables de la mise en œuvre de tels programmes aux échelles provinciale et locale. S'ils avaient accès plus facilement aux données de surveillance sur la maltraitance des enfants, les décideurs de la santé publique visés : 1) seraient davantage conscients des conclusions de l'ECI; 2) disposeraient de données statistiques pour pouvoir donner un caractère prioritaire à la maltraitance d'enfants comme problème de santé publique et, partant, acheminer en priorité des ressources à des programmes de prévention primaire; 3) seraient mieux informés des tendances en matière de maltraitance des enfants; 4) disposeraient de données sur les indicateurs de risque pouvant être modifiés au moyen d'interventions de santé publique; 5) pourraient mieux connaître les habitudes d'orientation des victimes vers des services d'aide à l'enfance par les professionnels de la santé publique; 6) auraient la possibilité de définir des priorités de recherches en matière de maltraitance des enfants; 7) seraient en mesure d'identifier les populations à cibler pour les interventions de prévention primaire.

À l'heure actuelle, on obtient grâce à l'ECI le meilleur instantané possible de l'incidence de la maltraitance d'enfants au Canada, mais ce système national de surveillance souffre de plusieurs limites. Premièrement, les résultats sont plus ou moins utiles, vu leur portée nationale et, donc, leur peu de validité à l'échelle locale du fait des techniques d'échantillonnage ${ }^{8}$. $\mathrm{Au}$ cours de chaque cycle, toutefois, il est loisible aux provinces et aux territoires d'obtenir une estimation de l'ampleur du problème de la maltraitance à leur échelle en prenant des dispositions pour qu'il y ait suréchantillonnage. Deuxièmement, le système de surveillance de l'ECI n'a pas été évalué de façon officielle, de sorte que l'on n'est pas certain de son efficacité quant à la cueillette, l'analyse et la communication des données. Troisièmement, pour planifier des programmes de prévention adaptés culturellement, il est impératif de recueillir des données exactes, particulièrement auprès des groupes considérés comme étant à risque. Or, l'ECI n'a pas encore obtenu d'échantillon représentatif des agences des Premières nations, encore que le nombre des agences participantes augmente de cycle en cycle, ce qui constitue un signe encourageant.

Quatrièmement - lacune la plus importante - les données de surveillance dépendent d'une source unique, les rapports des agences d'aide à l'enfance. Par conséquent, bien que l'ECI regorge de renseignements sur les enquêtes des agences d'aide à l'enfance, elle ne nous apprend rien sur les enquêtes de police réalisées sur les cas de maltraitance ou les cas non déclarés d'abus ou de négligence, de sorte qu'elle 
sous-estime le fardeau véritable de la maltraitance dans la population. Pour que l'on puisse bien comprendre l'état de la santé des enfants canadiens, ce qu'il faut, c'est un réseau de systèmes de surveillance de la santé publique arrimé à d'autres enquêtes et sources d'information sur la santé caractérisées par des éléments de données communs, des mécanismes de collecte et de distribution rapides de l'information et la facilité d'accès aux données ${ }^{12}$. Aux États-Unis, les Centers for Disease Control and Prevention mettent actuellement au point des systèmes alternatifs pour recueillir des renseignements dans les hôpitaux et les services d'urgence sur les cas mortels et les cas non mortels de maltraitance d'enfants et sur les victimes de violence, dans le but d'instituer un système national de renseignements sur les cas de mort violente ${ }^{13}$. Ces sources de données seront utilisées de concert avec les observations du National Child Abuse and Neglect Data System et de la National Incidence Study of Child Abuse and Neglect, qui comprend des sondages sentinelles auprès des professionnels locaux travaillant avec des enfants et des familles exclus du système de bien-être de l'enfance $^{14}$, afin de mieux jauger la maltraitance d'enfants. En Australie, un système regroupe des bases de données administratives sur les services de santé et les services sociaux offerts à tous les enfants, ce qui améliore la qualité des données recueillies sur la protection de l'enfance ${ }^{15}$. Au Canada, l'ASPC, ayant fondé l'ECI et étant chargée de sa coordination avec les autres systèmes de surveillance de la santé, est dans une position optimale pour coordonner l'établissement d'un système de surveillance intégré qui s'alimenterait à d'autres sources que les seuls rapports provenant des services d'aide à l'enfance.

\section{Identification des déterminants de la maltraitance des enfants}

Une multiplicité d'indicateurs de risque individuels, familiaux et locaux est associée à la violence physique, à l'exploitation sexuelle et à la négligence ${ }^{16}$. L'ECI fournit des renseignements sur les facteurs de risque grâce auxquels les chercheurs et les décideurs du domaine de la santé publique peuvent mener à bien des analyses secondaires afin de répondre à des questions pertinentes sur la maltraitance des enfants et d'établir des cadres conceptuels pouvant étayer l'élaboration de stratégies de prévention $^{5}$. Au nombre de ces facteurs de risque figurent les caractéristiques de l'enfant (âge, sexe, antécédents ethniques, etc.), les modalités de fonctionnement entre l'enfant et le gardien (exposition à la violence du partenaire intime, problèmes de toxicomanie, problèmes psychologiques, antécédents parentaux de mauvais traitements subis durant l'enfance, etc.) et les caractéristiques du ménage (structure, taille de la famille, revenu, emploi, etc.). Bien qu'elle ne vise pas à recueillir de données à l'échelon des collectivités, l'ECI fournit également certains renseignements sur les déterminants sociaux de la maltraitance d'enfants, tels les problèmes de logement, les faibles taux d'emploi et la pauvreté.

\section{Définition et élaboration de stratégies de prévention}

Il incombe aux départements de santé publique, par delà les secteurs de la santé et des services sociaux, de définir ou d'élaborer des interventions ou des programmes de prévention primaire pour lutter contre les problèmes de santé publique de base dans une perspective de santé des populations. La difficulté de cette tâche vient de ce que chaque catégorie particulière de maltraitance d'enfants est associée à des indicateurs de risque uniques qui, même s'ils partagent quelquefois des éléments communs, exigent des interventions sur mesure. Selon une étude récente sur les interventions visant la maltraitance d'enfants ${ }^{11}$, un seul programme de formation aux responsabilités parentales est apparu comme étant efficace pour prévenir la récidive de violence physique et aucune intervention n’a été jugée en mesure de prévenir la récidive de la négligence; seules quelques interventions se sont traduites par des améliorations du comportement ou de l'état psychologique des enfants qui avaient été victimes de négligence, exposés à la violence du partenaire intime ou fait l'objet de sévices sexuels. Un examen systématique des interventions de formation aux responsabilités parentales a révélé que des programmes complexes et polyvalents de visites à domicile ciblant des familles à risque avaient permis de prévenir efficacement les lésions non intentionnelles chez les enfants, une mesure indirecte de la négligence ${ }^{17}$. Deux examens de synthèse récents ont montré que le programme Partenariat infirmières-familles (PIF) représentait actuellement ce qu'il y a de mieux pour prévenir la maltraitance des enfants ${ }^{11,18}$ : des infirmières visitent à intervalles fréquents des femmes jeunes, à faible revenu, enceintes pour la première fois, depuis le moment de la grossesse (moins de 29 semaines de gestation) jusqu'à ce que l'enfant atteigne l'âge de deux ans. Trois essais aléatoires contrôlés (EAC) ont mis en évidence des effets bénéfiques multiples, constants et durables sur des paramètres relatifs à la santé de la mère et de l'enfant ${ }^{19-21}$. De plus, le programme PIF entraîne des économies de coûts importantes, les avantages économiques provenant vraisemblablement de la capacité du programme à améliorer les taux de réussite scolaire au niveau du secondaire et à aider les jeunes mères à se trouver un emploi, sans compter la réduction des taux de violence physique et de négligence, de toxicomanie, de criminalité et de recours à l'aide sociale ${ }^{22}$. Dans les évaluations, le programme PIF ressort constamment comme le programme par excellence en matière de ratio coûts avantages par participant, par comparaison avec la plupart des autres programmes de prévention ciblant les parents de nourrissons et de jeunes enfants aux États-Unis ${ }^{22-24}$.

À défaut de disposer d'interventions efficaces évaluées de façon stricte, les chercheurs du domaine de la santé publique peuvent se rabattre sur les facteurs de risque et de protection identifiés et les modèles théoriques établis pour élaborer et mettre à l'essai des interventions de prévention primaire. Mrazek et Haggerty ${ }^{25}$ ont mis au point un cadre intégré que plusieurs considèrent comme " le nec plus ultra » pour ce qui est de guider l'élaboration de ce type d'interventions. Le cadre, qui vient compléter les étapes de l'approche axée sur la santé publique, prévoit cinq grandes démarches : 1) identification et mesure du problème; 2) identification de facteurs de risque et de protection et de modèles théoriques provenant de nombreux domaines; 3) mise au point de l'intervention, formation des intervenants 
et exécution à petite échelle d'un projet pilote ou d'études de faisabilité débouchant sur un EAC reproduisant exactement l'intervention; 4) conduite à large échelle d'un EAC en vue de déterminer l'efficacité de l'intervention; 5) mise en œuvre générale de l'intervention et évaluation périodique du programme.

\section{Mise en œuvre et évaluation d'interventions et de politiques fondées sur des données probantes}

Tous les départements de santé publique devraient impérieusement s'employer, particulièrement lorsque les ressources à disposition sont limitées, à mettre en œuvre des interventions efficaces axées sur les familles à risque et à ne pas s'attarder à des programmes dont l'adéquation et l'efficacité n’ont pas été prouvées. Le programme PIF est reconnu sur le plan international comme étant l'intervention par excellence pour prévenir la maltraitance des enfants ${ }^{11}$. Très largement utilisé aux États-Unis, ce programme novateur est actuellement évalué et reproduit en Angleterre, en Écosse, en Allemagne, aux Pays-Bas et en Australie ${ }^{26}$. Le programme PIF est un exemple d'intervention de prévention primaire qui correspond à la pratique des infirmières en santé publique. Comme il incombe aux provinces et aux territoires de coordonner et de financer les services de santé directs, le programme devrait être mis en œuvre par un organisme de santé publique régional.

À l'heure actuelle, toutes les provinces et tous les territoires (à l'exception du Nunavut) offrent des programmes de visites à domicile. Certains ont un volet post-partum universel, mais tous s'adressent aux parents et aux ménages caractérisés par des indicateurs de risque synonymes de mauvais effets sur la santé et le développement de l'enfant ${ }^{27}$. Ces programmes sont principalement offerts par l'intermédiaire d'organismes de santé publique communautaires ou en collaboration avec eux. Tous ont les mêmes buts - promouvoir la croissance et le développement harmonieux de l'enfant en améliorant l'exercice des responsabilités parentales par la formation, l'orientation vers des services de proximité et des programmes de soutien social - mais tous ne sont pas identiques, variant considérablement par leurs objectifs, l'instruction et l'expérience des visiteuses à domicile (professionnelles, paraprofessionnelles ou non spécialistes), l'intensité et la fréquence des visites, le recours ou non à un cursus structuré, le moment de l'inscription au programme (pendant la grossesse ou pendant la période post-partum) et la durée de l'intervention. On ne dispose pas à l'heure actuelle de données objectives provenant d'évaluations provinciales ou territoriales et permettant de se faire une bonne idée des répercussions de ces stratégies de visites à domicile sur les paramètres relatifs à la santé de la mère et de l'enfant. De plus, la plupart des évaluations n’ont pas été réalisées conformément à un plan d'étude exigeant des comparaisons entre un groupe témoin et un groupe traité27, à l'exception de l'évaluation du programme Families First ${ }^{28}$. En l'absence de plans d'étude rigoureux, il est difficile de conclure que les interventions de visites à domicile sont à l'origine des changements observés.

Compte tenu du paradigme selon lequel les services de santé doivent reposer sur des données probantes, et au vu de la rareté fréquente des ressources dans le secteur de la santé publique, les départements de santé publique locaux ont le devoir moral de mettre en œuvre les interventions qui se sont avérées modifier de manière importante les principaux paramètres relatifs à la santé de la mère et de l'enfant, notamment celles qui peuvent prévenir la violence et la négligence. Toutefois, comme il se peut qu'un programme ne procure pas dans la pratique des avantages du même ordre que dans la théorie, il est impératif que la mise en œuvre soit précédée d'une étude pilote, puis d'une évaluation effectuée au moyen d'un $\mathrm{EAC}^{16,25}$. C'est cette approche que l'on utilise actuellement pour tester la faisabilité et l'acceptabilité du programme PIF dans le cadre des systèmes canadiens de soins de santé et de sécurité sociale. En 2008, les services de santé publique de la ville de Hamilton et une équipe pluridisciplinaire de chercheurs de l'Université McMaster ont adapté le cursus du programme pour pouvoir l'utiliser auprès de femmes jeunes, à faible revenu, enceintes pour la première fois et vivant à
Hamilton (Ontario). Ces chercheurs réalisent actuellement une étude pilote afin de tester les techniques de recrutement et les stratégies de rétention, de même que la possibilité de recueillir des données sur la maltraitance des enfants auprès d'organismes locaux d'aide à l'enfance, des données sur les hospitalisations des mères et des enfants, des données cliniques et des données d'entrevues avec des participantes. Ils effectuent également une étude qualitative afin de déterminer si cette intervention ciblée est acceptable pour les bénéficiaires et leur famille, les infirmières en santé publique chargées des visites à domicile et les professionnels locaux qui orientent les bénéficiaires et leur proposent des services de santé et de protection sociale secondaires. Si les résultats de ces études pilotes sont favorables, un EAC sera entrepris pour mesurer les répercussions et le ratio coûtsavantages du programme en Ontario et, éventuellement, dans d'autres communautés au Canada.

\section{Conclusion}

À titre de participant à une réponse plurisectorielle au problème de la maltraitance des enfants, le secteur de la santé publique - fort de ses solides fondations - fait aujourd'hui preuve de leadership, mesure l'ampleur du problème, définit les indicateurs de risque individuels, familiaux et communautaires de la maltraitance et délimite, met en œuvre et évalue des stratégies de prévention primaire. Ses prochaines priorités devraient être : 1) de communiquer dans un délai raisonnable les données de surveillance de l'ECI aux décideurs responsables de la mise en œuvre des programmes de prévention des blessures chez les enfants et les jeunes ou des programmes de formation aux responsabilités parentales au niveau provincial et territotial; 2) de solliciter la collaboration de chercheurs en santé publique pour qu'ils effectuent des analyses secondaires des données de l'ECI afin de répondre aux questions pertinentes; 3) de mettre en place un processus d'évaluation de l'ECI.

Le raffinement des données de surveillance de l'ECI et leur communication 
TABLEAU 1

Réponses des secteurs à la maltraitance des enfants

\begin{tabular}{|c|c|}
\hline Secteur & Réponse globale à la maltraitance des enfants \\
\hline 1. Aide à l'enfance & $\begin{array}{l}\text { - } \quad \text { Donne suite aux cas observés ou présumés de mauvais traitement et fait enquête au besoin } \\
\text { - } \quad \text { Offre du counseling et des services aux contrevenants, aux enfants à risque et aux enfants maltraités } \\
\text { - } \quad \text { Prévient la répétition des actes de maltraitance (prévention secondaire) }\end{array}$ \\
\hline 2. Justice & $\begin{array}{l}\text { - } \quad \text { Réforme juridique } \\
\text { - } \quad \text { Poursuite des contrevenants } \\
\text { Protection des enfants témoins }\end{array}$ \\
\hline 3. Enseignement & $\begin{array}{l}\text { - Montre aux élèves et aux parents comment reconnaître les différents types de mauvais traitements, comment } \\
\text { les prévenir et y réagir }\end{array}$ \\
\hline $\begin{array}{l}\text { 4. Services de soins de santé primaires } \\
\text { et de soins de santé de courte durée }\end{array}$ & $\begin{array}{l}\text { - Identification et documentation des signes et des symptômes de mauvais traitements } \\
\text { - } \quad \text { Évaluation des enfants exposés à de mauvais traitements } \\
\text { Traitement des lésions physiques ou des traumatismes psychologiques causés par des mauvais traitements } \\
\text { (prévention tertiaire) }\end{array}$ \\
\hline 5. Services de police & $\begin{array}{l}\text { - Donnent suite aux cas observés ou présumés de mauvais traitements, font enquête au besoin et déterminent } \\
\text { s'il y a infraction pénale } \\
\text { - Procèdent à l'identification et à l'arrestation des contrevenants allégués } \\
\text { - Déposent, le cas échéant, des accusations appropriées au criminel }\end{array}$ \\
\hline 6. Santé publique & $\begin{array}{l}\text { - } \quad \text { Coordonne les systèmes de surveillance, y compris la collecte, l'analyse et la diffusion des données } \\
\text { - } \quad \text { Définit, met en œeuvre et évalue des programmes de prévention primaire }\end{array}$ \\
\hline
\end{tabular}

subséquente aux décideurs du domaine de la santé publique sensibilisera ces derniers aux indicateurs de risque liés à la maltraitance des enfants, ce qui permettra d'accorder la priorité à la mise en œuvre d'interventions visant ces indicateurs. Une masse importante d'études désignent le programme PIF comme la méthode par excellence en termes de coûts pour prévenir la maltraitance des enfants et améliorer d'autres paramètres importants relatifs à la mère et à l'enfant. Au terme d'une évaluation appropriée, les départements de santé publique pourraient donc mettre en œuvre le programme PIF afin de consolider les programmes universels actuels de formation aux responsabilités parentales.

Avec l'apparition de programmes de prévention dont efficacité est notoire, les chercheurs et les décideurs du domaine de la santé publique doivent continuer à favoriser une approche plurisectorielle et des ressources permettant une évaluation rigoureuse des programmes. Comme pour toute autre intervention en matière de soins de santé, nous devrions être capables d'expliquer clairement aux bénéficiaires les avantages, et les préjudices éventuels, que leur consentement à participer à un programme de santé publique leur apporterait.

\section{Remerciements}

Mme Jack a bénéficié, pour la préparation de ce commentaire, du concours de la Section des blessures et de la violence envers les enfants de l'Agence de la santé publique du Canada. Elle détient une bourse de personnel de recherche de l'Institut du développement et de la santé des enfants et des adolescents, Santé de la reproduction et de l'enfant, des Instituts de recherche en santé du Canada. Merci à Lil Tonmyr et à Wendy Hovdestad, de la Section des blessures et de la violence envers les enfants, Division de la surveillance de la santé et de l'épidémiologie, Agence de la santé publique du Canada, pour leurs observations concernant les premières ébauches de ce manuscrit.

\section{Références}

1. Leeb RT, Paulozzi LJ, Melanson C, Simon TR, Arias I. Child maltreatment surveillance: uniform definitions for public health and recommended data elements. Atlanta (GA): Centers for Disease Control and Prevention, National Center for Injury Prevention and Control; 2008 Jan.

2. Cicchetti D, Toth SS. Child maltreatment. Annu Rev Clin Psychol. 2005;1:409-38.

3. MacMillan HL, Munn C. The sequelae of child maltreatment. Curr Opin Psychiatr. 2001;14(4):325-31.

4. MacMillan HL, Fleming JE, Trocme N, Boyle MH, Wong M, Racine YA, Beardslee WR, Offord DR. Prevalence of child physical and sexual abuse in the community: results from the Ontario Health Supplement. JAMA. 1997;278(2):131-5.

5. Guyer B. Problem-solving in public health. Dans : Armenian HK, Shapiro S, dirs. Epidemiology and health services. New York: Oxford University Press; 1998. p. 15-26. 
6. Hammond WR. Public health and child maltreatment prevention: the role of the Centers for Disease Control and Prevention. Child Maltreat. 2003; 8(2):81-3.

7. Krug EG, Dahlberg LL, Mercy JA, Zwi AB, Lozano R, dirs. Rapport mondial sur la violence et la santé. Genève : Organisation mondiale de la santé; 2002.

8. Trocmé N, Fallon B, MacLaurin B, Daciuk J, Felstiner C, Black T, Tonmyr L, Blackstock C, Barter K, Turcotte D, Cloutier R. Étude canadienne sur l'incidence des signalements de cas de violence et de négligence envers les enfants - 2003 : Données principales. Ottawa : Ministère des Travaux publics et des Services gouvernementaux; 2005.

9. Pless B. Surveillance alone is not the answer. Inj Prev. 2008;14(4):220-2.

10. Jack S, Tonmyr L. Knowledge transfer and exchange: disseminating Canadian child maltreatment surveillance findings to decision makers. Child Ind Res. 2008;1(1):51-64.

11. MacMillan HL, Wathen $\mathrm{CN}$, Barlow J, Fergusson DM, Leventhal JM, Taussig HN. Interventions to prevent child maltreatment and associated impairment. Lancet. 2009;373(9659):250-66.

12. Thacker SB, Stroup DF. Public health surveillance and health services research. In: Armenian HK, Shapiro S, editors. Epidemiology and health services. New York: Oxford University Press; 1998. p. 61-82.

13. Whitaker DJ, Lutzker JR, Shelley GA. Child maltreatment prevention priorities at the Centers for Disease Control and Prevention. Child Maltreat. 2005;10(3):245-59.

14. Sedlak AJ, Broadhurst DD. Executive summary of the third national incidence study of child abuse and neglect. Washington (DC): US Department of Health and Human Services, Administration for Children and Families, Administration on Children, Youth and Families, National Center on Child Abuse and Neglect;1996.
15. Fluke J, Tonmyr L, Gray J, Bianchi D, Halifax J, Kim C. The ISPCAN working group on Data Collection. Frameworks for international comparison of child maltreatment data. In: World Perspectives on Child Abuse, 8 éd. Daro D, dir. West Chicago (IL): International Society for Prevention of Child Abuse and Neglect; 2008. p. 48-66

16. Gonzalez A, MacMillan H. Preventing child maltreatment: an evidence-based update. J Postgrad Med. 2008;54(4):280-6.

17. Kendrick D, Barlow J, Hampshire A, Polnay L, Stewart-Brown S. Parenting interventions for the prevention of unintentional injuries in childhood. Cochrane Database of Systematic Reviews. 2007, 4. Art. $\mathrm{n}^{\circ}$ CD006020. DOI: 10.1002/14651858. CD006020.pub2

18. Gomby DS. Home visitation in 2005: outcomes for children and parents. Washington (DC): Committee for Economic Development, Invest in Kids Working Group; 2005 Jul 18.

19. Kitzman H, Olds DL, Henderson CR Jr, Hanks C, Cole R, Tatelbaum R, McConnochie KM, Sidora K, Luckey DW, Shaver D, Engelhardt K, James D, Barnard K. Effect of prenatal and infancy home visitation by nurses on pregnancy outcomes, childhood injuries, and repeated childbearing. A randomized controlled trial. JAMA. 1997;278(8):644-52.

20. Olds DL, et al. Home visiting by paraprofessionals and by nurses: a randomized, controlled trial. Pediatrics. 2002;110(3):486-96.

21. Olds DL et al. Long-term effects of home visitation on maternal life course and child abuse and neglect. Fifteen-year follow-up of a randomized trial. JAMA. 1997;278(8);637-43.

22. Jones D, Bumbarger BK, Greenberg $\mathrm{M}$, Greenwood P, Kyler S. The economic return of PCCD's investment in researchbased programs: a cost-benefit assessment of delinquency prevention in Pennsylvania [Internet]. Pennsylvania State University (PA): Prevention Research Center for the Promotion of Human Development; 2008
Mar [consultation le $1^{\text {er }}$ mars 2009]. PDF (1,71 Mo) téléchargeable à partir du lien : http://prevention.psu.edu/pubs/docs/ PCCD_Report2.pdf

23. Lee S, Aos S, Miller M. Evidence-based programs to prevent children from entering and remaining in the child welfare system: benefits and costs for Washington [Internet]. Olympia (WA): Washington State Institute for Public Policy; 2008 Jul [consultation le $1^{\text {er }}$ mars 2009]. PDF (382 Ko) téléchargeable à partir du lien : http://www.wsipp.wa.gov/rptfiles/ 08-07-3901.pdf

24. McGroder SM, Hyra A. Developmental and economic effects of parenting programs for expectant parents and parents of preschool-age children [Internet]. Washington (DC): Partnership for America's Economic Success; 2009 Feb 18 [consultation le $1^{\text {er }}$ mars 2009]. PDF (1,06 Mo) téléchargeable à partir de la page : http://www. partnershipforsuccess.org/docs/researchproject_mcgroder_200903_paper.pdf

25. Mrazek PJ, Haggerty RJ, editors. Reducing risks for mental disorders: Frontiers for preventive intervention research. Washington (DC): National Academy Press; 1994.

26. Prevention Research Center for Family and Child Health. Nurse-Family Partnership international program. [Internet]. Denver (CO): University of Colorado Department of Pediatrics; n.d. [consultation le 18 août 2010]. Consultable à la page : http://www. ucdenver.edu/ACADEMICS/COLLEGES/ MEDICALSCHOOL/DEPARTMENTS/ PEDIATRICS/RESEARCH/PROGRAMS/ PRC/RESEARCH/INTERNATIONAL/Pages/ international.aspx.

27. Petitclerc A. An overview of home-visiting services across Canada. Early Childhood Learning Knowledge Centre Bulletin. 2008 March;3(1):5-8.

28. Enfants en santé Manitoba. Les familles d'abord : points saillants de l'évaluation du programme. Winnipeg (Man.) : Enfants en santé Manitoba; 2010 [consultation le 5 juillet 2010]. Consultable en ligne à partir de la page : http://www.gov.mb.ca/ 